\title{
Predicting survival among high-risk pediatric cardiac transplant recipients: An analysis of the United Network for Organ Sharing database
}

Ryan R. Davies, MD, ${ }^{a}$ Mark J. Russo, MD, MS, ${ }^{\mathrm{b}}$ Seema Mital, MD, ${ }^{\mathrm{c}}$ Timothy M. Martens, MD, ${ }^{a}$ Robert S. Sorabella, $\mathrm{BA}^{\mathrm{a}}$ Kimberly N. Hong, MHSA, ${ }^{\text {b }}$ Annetine C. Gelijns, PhD, ${ }^{\text {b }}$ Alan J. Moskowitz, MD, Jan M. Quaegebeur, MD, ${ }^{a}$ Ralph S. Mosca, MD, and Jonathan M. Chen, MD ${ }^{d}$

Supplemental material is available online.
From the Division of Cardiothoracic Surgery $^{\mathrm{a}}$ and the International Center for Health Outcomes and Innovation Research, ${ }^{\mathrm{b}}$ Department of Surgery, Children's Hospital of New York-Presbyterian and Columbia University College of Physicians and Surgeons, New York, NY; the Division of Pediatric Cardiology, ${ }^{\mathrm{c}}$ Hospital for Sick Children, Toronto, Ontario, Canada; and Department of Cardiothoracic Surgery, ${ }^{\mathrm{d}}$ Weill Medical College of Cornell University, New York, NY.

This work was funded in part by the Health Resources and Services Administration contract 231-00-0115 and departmental funding sources.

The content of this article is the responsibility of the authors alone and does not necessarily reflect the views or policies of the Department of Health and Human Services, nor does mention of trade names, commercial products, or organizations imply endorsement by the US Government.

Read at the Thirty-third Annual Meeting of the Western Thoracic Surgical Association, Santa Ana Pueblo, NM, June 27-30, 2007.

Received for publication July 3, 2007; revisions received Aug 21, 2007; accepted for publication Sept 6, 2007.

Address for reprints: Jonathan M. Chen, MD, Pediatric Cardiac Surgery, Children's Hospital of New York, 3959 Broadway, Suite 2-273, New York, NY 10032 (E-mail: jmc23@columbia.edu).

J Thorac Cardiovasc Surg 2008;135:147-55 0022-5223/\$34.00

Copyright (C) 2008 by The American Association for Thoracic Surgery

doi:10.1016/j.jtcvs.2007.09.019
Objective: Studies of high-risk pediatric cardiac transplant recipients are lacking. The purpose of this study is to evaluate early posttransplant survival in high-risk pediatric patients.

Methods: The United Network for Organ Sharing (UNOS) provided de-identified patient-level data. The study population included 3502 recipients aged less than 21 years who underwent transplantation from January 1, 1995, through December 31, 2005. Recipients were stratified on the basis of the presence or absence of high-risk criteria: pulmonary vascular resistance index greater than 6 Wood units $/ \mathrm{m}^{2}(\mathrm{n}=$ $285,8.1 \%$ ), creatinine clearance less than $40 \mathrm{~mL} / \mathrm{min}(308,8.8 \%)$, hepatitis C positivity $(33,0.9 \%)$, donor/recipient weight ratio less than $0.7(80,2.3 \%)$, panel reactive antibody greater than $40 \%(235,6.7 \%)$, retransplantation $(235,6.7 \%)$, and age less than 1 year old $(840,24.0 \%)$.

Results: Overall, 1575 (45.0\%) patients met at least one high-risk criterion. Higher numbers of high-risk criteria in a patient were correlated with increased 30-day mortality (0 high-risk criteria: $5.2 \% ; 1$ criterion: $7.9 \% ; 2$ criteria: $12.9 \%$; and 3 or more criteria: $25.0 \% ; P<.0001)$ and poor long-term survival $(P<.0001)$. Among patients with high-risk criteria, a simplified scoring scale accurately predicts both 30 -day and contingent 1-year mortality $(P<.0001)$.

Conclusions: Individually, the effect of high-risk criteria on posttransplant survival varied; however, increasing numbers of criteria in a patient resulted in a cumulative increase in mortality. A scoring scale allows for the prediction of approximate mortality rates after transplantation. These findings suggest that recipient criteria for transplantation should focus on the number of high-risk criteria as well as clinical status, rather than the presence or absence of a single risk factor.

$\mathrm{O}$ ptimal allocation of the limited — and decreasing ${ }^{1}$ — supply of pediatric donor hearts requires accurate pretransplant assessment of survival and data-derived selection of criteria indicating high risk. Unfortunately, criteria for transplant eligibility remain largely the result of consensus opinion.

In the adult population, continued improvements in posttransplant outcomes (as well as the ongoing shortage of donor organs) have prompted attempts to expand the pools of both donors and recipients. Results from the use of such "alternate-list" strategies have been variable. Some authors have reported results equivalent to those in standard criteria receipients, ${ }^{2}$ whereas others have had less success. ${ }^{3}$ In some cases, specific traditional high-risk criteria (HRC) have failed to consistently predict poor posttransplant outcome (older age, ${ }^{4-6}$ recipient hepatitis $C$ positivity ${ }^{7}$ ), whereas others clearly increase posttransplant mortality (elevated pulmonary vascular resistance $[\mathrm{PVR}]^{8}$ ). 
The applicability of any of these data to pediatric recipients is unclear. Although less well studied in children, some results suggest that pediatric HRC recipients have acceptable outcomes and that our criteria remain too strict. ${ }^{9,10}$ Evidence-based estimates of posttransplant survival in a pediatric population would allow for better stratification of potential recipients and optimization of transplant selection criteria.

This report uses data from the United Network for Organ Sharing (UNOS) database to assess posttransplant outcomes in patients meeting traditional HRC. Our goals were (1) to estimate the impact of HRC on posttransplant outcomes and (2) to develop a simplified scoring system to predict shortterm posttransplant survival.

\section{Methods}

\section{Data Collection}

UNOS provided de-identified patient level data from the Thoracic Registry (data source \#021606-4). Use of these data is consistent with the regulations of our university's institutional review board. Records with incomplete data were excluded from analyses requiring those data points.

\section{Study Population}

The study population consists of 3502 transplants performed on patients less than 21 years of age between January 1, 1995, and December 31, 2005. Recipients were stratified on the basis of the presence or absence of traditional HRC: PVR index greater than 6 Wood units $/ \mathrm{m}^{2}(\mathrm{n}=285,8.1 \%)$, creatinine clearance $(\mathrm{CrCl})$ less than $40 \mathrm{~mL} / \mathrm{min}(308,8.8 \%)$, hepatitis C positivity $(33,0.9 \%)$, donor/ recipient weight ratio less than $0.7(80,2.3 \%)$, panel reactive antibody greater than $40 \%(235,6.7 \%)$, retransplantation $(235,6.7 \%)$, and age less than 1 year old $(840,24.0 \%)$. These criteria were established before data analysis and are based on internal criteria for transplantation at our institution, as well as a review of published reports. ${ }^{11}$ Congenital heart disease (CHD) was not considered as one of the HRC because previous work had suggested that it is complex rather than any CHD which indicated elevated risk, ${ }^{9}$ and the UNOS database does not contain detailed data regarding the CHD diagnosis that would enable such stratification. In addition, patients were grouped by the number of HRC present: one $(\mathrm{n}=1189,34.0 \%)$, two $(\mathrm{n}=334$, $9.5 \%)$, and three or more $(\mathrm{n}=52,1.5 \%)$ Overall, $1575(45.0 \%)$ patients met at least one HRC.

\section{Data Analysis}

Data were analyzed by SAS 9.13 for Windows software (SAS Institute, Inc, Cary, NC). The primary outcomes were 30-day and contingent (on 30-day survival) 1-year mortality; other outcomes included long-term survival (time to death) and in-hospital complications. All variables analyzed are available in Table E1; only significant variables are reported. Continuous variables are reported as means \pm standard deviation and were compared by the Student $t$ test (with Bonferroni correction). Ordinal variables were compared by the $\chi^{2}$ test. All $P$ values are 2 -sided. Multivariate regression (stepwise, $P<.05$ ) was also performed. Kaplan-Meier analysis and Cox proportional hazards regression (stepwise, $P<.05$ ) were used for time-to-event analysis; patients without accurate follow-up times were excluded from these analyses. Risk, odds (OR), and hazard ratios are reported with 95\% confidence intervals $(95 \% \mathrm{CI})$ in parentheses. Simplified predictive scores were developed for both 30-day and contingent 1-year mortality. Data from both multivariate and univariate analysis were used to assign points on the basis of the presence of specific comorbidities. The predictive value of the scores was assessed by the $\chi^{2}$ test and logistic regression.

\section{Results}

Baseline demographics are given in Table 1 with a comparison of HRC versus normal-risk recipients; patients in the HRC group required higher levels of support and critical medical care before transplantation.

\section{Early and Late Mortality}

Thirty-day $(9.2 \%$ vs $5.2 \% ; P<.0001)$ and contingent 1 -year mortality $(10.2 \%$ vs $7.7 \% ; P<.0229)$ were higher in patients with HRC than in those without. Within the HRC group, indicators of clinical support (hospitalization, mechanical ventilation) were the most significant predictors of 30-day and contingent 1-year mortality (Table 2). Patients undergoing retransplantation and those with elevated panel reactive antibody or PVR index did not have an increased risk of early mortality (Table 2), whereas those with renal failure did (OR 3.01, 95\% CI 2.05-4.43). Small sample sizes limited the analysis of hepatitis $\mathrm{C}$ positivity $(\mathrm{n}=33$, OR $1.72,95 \% \mathrm{CI}$ 0.65-4.52) and low donor/recipient weight ratio $(\mathrm{n}=80$, OR $1.77,95 \%$ CI $0.94-3.36)$. Later year of transplantation was associated with increased survival at 30 days $(P=.0069)$. In multivariate analysis, similar factors were important in predicting poor short-term outcomes (Table 3).

\section{In-hospital Complications}

Early survival was drastically reduced in HRC patients having postoperative complications. Patients requiring dialysis had significantly higher 30 -day $(28.0 \%$ vs $5.2 \%$ ) (OR 7.10, 95\% CI 5.13-9.81) and contingent 1 -year mortality (38.4\% vs $7.2 \%$ ) (OR 8.04, 95\% CI 5.52-11.71). Less severe increases in 30-day (10.7\% vs $5.4 \%$ ) (OR $2.10,95 \%$ CI $1.59-2.79)$ and contingent 1-year mortality (17.4\% vs 6.3\%) (OR 3.14, 95\% CI 2.38-4.16) occurred in patients with postoperative infections.

Multivariate predictors of the need for postoperative dialysis in $\mathrm{HRC}$ patients included preoperative $\mathrm{CrCl}$ less than $40 \mathrm{~mL} / \mathrm{min}$ (OR $4.03,95 \%$ CI $2.54-6.42)$, redo transplantation (OR 2.32, 95\% CI 1.28-4.18), and the need for mechanical ventilation (OR 2.54, 95\% CI 1.57-4.09); age less than 1 year was protective (OR $0.56,95 \%$ CI $0.34-$ 0.94). Infections occurred with greater frequency in patients with poor pretransplant clinical status as indicated by $\mathrm{CrCl}$ less than $40 \mathrm{~mL} / \mathrm{min}$ (OR 1.44, 95\% CI 1.02-2.03), the need for mechanical ventilation (OR 2.47, 95\% CI 1.74-3.50), intensive care support (OR 1.59, 95\% CI 1.11-2.26), as well 
TABLE 1. Baseline demographics*

\begin{tabular}{|c|c|c|c|c|}
\hline \multirow[b]{2}{*}{ Variable } & \multirow[b]{2}{*}{ Overall $(\mathrm{n}=3502)$} & \multicolumn{3}{|c|}{ Standard vs high-risk group } \\
\hline & & Standard $(n=1927)$ & High risk $(n=1575)$ & $P$ value \\
\hline Gender (male) & $1987(56.7 \%)$ & $1106(57.4 \%)$ & $881(55.9 \%)$ & .3862 \\
\hline \multicolumn{5}{|l|}{ Age } \\
\hline $0-1$ y & $1092(24.0 \%)$ & $0(0.0 \%)$ & $840(53.3 \%)$ & $<.0001$ \\
\hline $2-5$ y & $703(20.1 \%)$ & $465(24.1 \%)$ & $238(15.1 \%)$ & $<.0001$ \\
\hline $6-12$ y & $677(19.3 \%)$ & $468(24.3 \%)$ & $209(13.2 \%)$ & $<.0001$ \\
\hline $13-18$ y & $934(26.7 \%)$ & $720(37.4 \%)$ & $214(13.6 \%)$ & $<.0001$ \\
\hline $19-21$ y & $348(9.9 \%)$ & $274(14.2 \%)$ & $74(4.7 \%)$ & $<.0001$ \\
\hline $\mathrm{BSA}<1.00 \mathrm{~m}^{2}$ & $1811(53.2 \%)$ & $656(35.1 \%)$ & $1155(75.3 \%)$ & $<.0001$ \\
\hline \multicolumn{5}{|l|}{ Etiology of heart failure } \\
\hline CHD & $1427(40.8 \%)$ & $657(34.1 \%)$ & $770(48.9 \%)$ & $<.0001$ \\
\hline Dilated cardiomyopathy & $1483(42.4 \%)$ & $1014(52.6 \%)$ & $469(29.8 \%)$ & $<.0001$ \\
\hline Hypertrophic cardiomyopathy & $82(2.3 \%)$ & $58(3.0 \%)$ & $24(1.5 \%)$ & .0038 \\
\hline \multicolumn{5}{|l|}{ Medical history } \\
\hline Previous cardiac operation & $652(48.5 \%)$ & $346(46.6 \%)$ & $306(50.8 \%)$ & .1255 \\
\hline History of stroke & $108(3.1 \%)$ & $60(3.2 \%)$ & $48(3.0 \%)$ & .8580 \\
\hline \multicolumn{5}{|l|}{ Support at transplantation } \\
\hline Inotropes & $1809(51.7 \%)$ & $971(50.4 \%)$ & $838(53.2 \%)$ & .0970 \\
\hline Transfusion since listing & $989(31.9 \%)$ & $388(22.8 \%)$ & $601(42.9 \%)$ & $<.0001$ \\
\hline Hospitalized & $2328(66.7 \%)$ & $1189(62.0 \%)$ & $1139(72.5 \%)$ & $<.0001$ \\
\hline In intensive care unit & $1900(54.5 \%)$ & $913(4.6 \%)$ & $987(62.8 \%)$ & $<.0001$ \\
\hline Mechanical ventilation & $592(16.9 \%)$ & $191(9.9 \%)$ & $401(25.5 \%)$ & $<.0001$ \\
\hline \multicolumn{5}{|l|}{ Mechanical circulatory support } \\
\hline Any type & $519(14.8 \%)$ & $311(16.1 \%)$ & $208(13.2 \%)$ & .0151 \\
\hline Intra-aortic balloon pump & $43(1.2 \%)$ & $26(1.4 \%)$ & $17(1.1 \%)$ & .4706 \\
\hline ECMO & $186(5.3 \%)$ & $78(4.1 \%)$ & $108(6.9 \%)$ & .0002 \\
\hline Ventricular assist device & $316(9.0 \%)$ & $221(11.5 \%)$ & $95(6.0 \%)$ & $<.0001$ \\
\hline
\end{tabular}

$B S A$, Body surface area; $C H D$, congenital heart disease; ECMO, extracorporeal membrane oxygenation. *Total number of patients varies for each row due to incomplete data entry. Numbers in parentheses reflect prevalence of positive response among those in whom the variable was entered.

as in younger patients between 2 and 5 years old (OR 1.55, 95\% CI 1.06-2.26) and those with CHD (OR 1.38, 95\% CI $1.03-1.86)$.

\section{Impact of Multiple HRC}

An increasing number of HRC present in a single patient resulted in cumulative higher mortality, particularly within the first year (Figure 1). Cox regression analysis of patients within the HRC group demonstrated that the need for extracorporeal membrane oxygenation (ECMO) (hazard ratio 2.26, 95\% CI 1.60-3.18), $\mathrm{CrCl}$ less than $40 \mathrm{~mL} / \mathrm{min}$ (hazard ratio $1.73,95 \%$ CI $1.37-2.20$ ), the presence of three or more HRC (hazard ratio $2.05,95 \%$ CI 1.33-3.17), and age between 13 and 18 years old (hazard ratio 1.59, 95\% CI 1.18-2.14) were all associated with poor long-term survival.

\section{Predicting Survival in High-risk Candidates}

Significant univariate and multivariate predictors of mortality at 30 days and 1 year were used to construct a predictive score with points awarded on the basis of the OR for mortality associated with each predictor (Table 4) (see
Appendix E1 for details of score construction). The most accurate predictive score based on receiver operating characteristic curves (Figure E1) is shown. Higher risk scores were associated with an increasing risk of both 30-day and overall (noncontingent) 1 -year mortality $(P<.0001)$ (Figure 2). Evaluation of the mortality associated with each score demonstrated three categories of risk: low, moderate, and high. Overall 30-day and 1-year mortality for each risk category are shown in Figure 3.

\section{Discussion}

Allocation of the limited number of pediatric cardiac allografts remains controversial: should an available organ be given to the patient most likely to die without the transplant or the one most likely to survive after transplantation? Alternate lists for transplantation might provide a system for maximizing the equitability of the waiting list, but providing marginal organs for the sickest patients may worsen the problem. Therefore, optimization of organ allocation requires accurate assessments of posttransplant survival and accurate delineation of a 
TABLE 2. Univariate predictors of 30-day and contingent 1-year mortality within the high-risk recipients

\begin{tabular}{|c|c|c|c|c|c|c|}
\hline \multirow[b]{2}{*}{ Risk factor } & \multicolumn{3}{|c|}{ Thirty-day mortality } & \multicolumn{3}{|c|}{ Contingent 1-year mortality } \\
\hline & $\mathbf{O R}$ & $95 \% \mathrm{Cl}$ & $P$ value & $\mathbf{O R}$ & $95 \% \mathrm{Cl}$ & $P$ value \\
\hline \multicolumn{7}{|l|}{ High-risk criteria } \\
\hline Renal failure $(\mathrm{CrCl}<40 \mathrm{~mL} / \mathrm{min})$ & 3.01 & $2.05-4.43$ & $<.0001^{*}$ & 2.28 & $1.47-3.53$ & $.0002^{*}$ \\
\hline Donor/recipient weight ratio $<0.7$ & 1.77 & $0.94-3.36$ & .0754 & 0.94 & $0.40-2.23$ & .8856 \\
\hline Hepatitis C & 1.72 & $0.65-4.52$ & .2657 & 1.88 & $0.63-5.63$ & .2499 \\
\hline Infant $(<1$ year old $)$ & 1.35 & $0.96-1.90$ & .0853 & 1.05 & $0.72-1.52$ & .8117 \\
\hline Elevated PRA & 1.09 & $0.69-1.73$ & .7058 & 0.79 & $0.44-1.41$ & .4205 \\
\hline Retransplantation & 0.81 & $0.49-1.34$ & .4153 & 0.81 & $0.46-1.42$ & .4563 \\
\hline Elevated PVR (>6 Wood units) & 0.74 & $0.37-1.48$ & .3934 & 1.01 & $0.52-1.95$ & .9761 \\
\hline \multicolumn{7}{|l|}{ No. of high-risk criteria } \\
\hline 1 & 0.51 & $0.36-0.72$ & $.0001^{*}$ & 0.76 & $0.50-1.15$ & .1933 \\
\hline 2 & 1.57 & $1.07-2.28$ & $.0188^{*}$ & 1.31 & $0.85-2.03$ & .2257 \\
\hline$\geq 3$ & 3.37 & $1.76-6.47$ & $.0001^{*}$ & 1.22 & $0.42-3.53$ & .7116 \\
\hline \multicolumn{7}{|l|}{ Etiology of heart failure } \\
\hline $\mathrm{CHD}$ & 1.98 & $1.40-2.81$ & $<.0001^{*}$ & 1.70 & $1.16-2.50$ & $.0058^{*}$ \\
\hline Dilated cardiomyopathy & 0.40 & $0.25-0.63$ & $<.0001^{*}$ & 0.53 & $0.33-0.84$ & $.0060^{*}$ \\
\hline \multicolumn{7}{|l|}{ Support at transplantation } \\
\hline ECMO & 3.86 & $2.42-6.16$ & $<.0001^{*}$ & 2.14 & $1.11-4.14$ & $.0202^{*}$ \\
\hline Mechanical ventilation & 2.05 & $1.45-2.91$ & $<.0001^{*}$ & 1.86 & $1.25-2.77$ & $.0020^{*}$ \\
\hline Intensive care unit & 1.91 & $1.30-2.82$ & $.0008^{*}$ & 1.69 & $1.12-2.54$ & $.0119 *$ \\
\hline Hospitalization & 1.74 & $1.13-2.66$ & $.0105^{*}$ & 1.79 & $1.13-2.86$ & $.0126^{*}$ \\
\hline Transfusion since listing & 1.68 & $1.17-2.40$ & $.0046^{*}$ & 2.06 & $1.37-3.10$ & $.0004^{*}$ \\
\hline Intra-aortic balloon pump & 0.59 & $0.08-4.48$ & 6071 & 3.00 & $0.95-9.45$ & $.0489 *$ \\
\hline \multicolumn{7}{|l|}{ Pretransplant medical history } \\
\hline Previous cardiac surgery & 2.12 & $1.18-3.82$ & $.0105^{*}$ & 2.84 & $1.45-5.55$ & $.0016^{*}$ \\
\hline Stroke & 1.65 & $0.73-3.76$ & .2231 & 3.05 & $1.34-6.96$ & $.0053^{*}$ \\
\hline Ischemic time $>4 \mathrm{~h}$ & 1.59 & $1.11-2.29$ & $.0115^{*}$ & 1.34 & $0.90-1.99$ & .1490 \\
\hline Age $6-12$ y & 0.39 & $0.20-0.78$ & $.0058^{*}$ & 0.69 & $0.38-1.26$ & .2253 \\
\hline
\end{tabular}

$O R$, Odds ratio; $C l$, confidence interval; $C r C l$, creatinine clearance; $P R A$, panel reactive antibody; $P V R$, pulmonary vascular resistance; $C H D$, congenital heart disease; ECMO, extracorporeal membrane oxygenation. * Significant at $P<0.05$ level.

high-risk group unsuitable for transplantation with standard risk donor organs.

In adults, alternate-list strategies have resulted in acceptable outcomes with suboptimal donor organs, including similar rates of primary graft dysfunction ${ }^{12}$ and only slightly decreased survival ${ }^{13}$ compared with standard recipient/donor pairs. Expansion of such policies to the pediatric population may result in a more optimal distribution of organs. The present study analyzes a set of traditional HRC to examine their impact on early mortality after cardiac transplantation in a pediatric population and to develop a more accurate pretransplant assignment of patients to posttransplant risk groups.

\section{Are HRC Truly High Risk?}

We found that several criteria traditionally used to identify "high-risk" patients had poor correlation with early transplant mortality. Elevated PVR index was not associated

TABLE 3. Multivariate predictors of 30 -day and contingent 1-year mortality within the high-risk recipients

\begin{tabular}{|c|c|c|c|c|c|c|}
\hline & \multicolumn{3}{|c|}{ Thirty-day mortality } & \multicolumn{3}{|c|}{ Contingent 1-year mortality } \\
\hline & OR & $95 \% \mathrm{CI}$ & $P$ value & $\mathbf{O R}$ & $95 \% \mathrm{CI}$ & $P$ value \\
\hline ECMO & 3.94 & $2.26-6.86$ & $<.0001$ & 2.10 & $1.04-4.26$ & .0387 \\
\hline No. of $H R C \geq 3$ & 2.42 & $1.09-5.36$ & .0302 & & & \\
\hline CHD & 1.64 & $1.08-2.48$ & .0207 & 1.68 & $1.10-2.57$ & .0158 \\
\hline Year of transplant & 0.91 & $0.85-0.97$ & .0036 & & & \\
\hline
\end{tabular}

$O R$, Odds ratio; $\mathrm{Cl}$, confidence interval; $E C M O$, extracorporeal membrane oxygenation; $\mathrm{CrCl}$, creatinine clearance; $H R C$, high-risk criteria; $C H D$, congenital heart disease. 


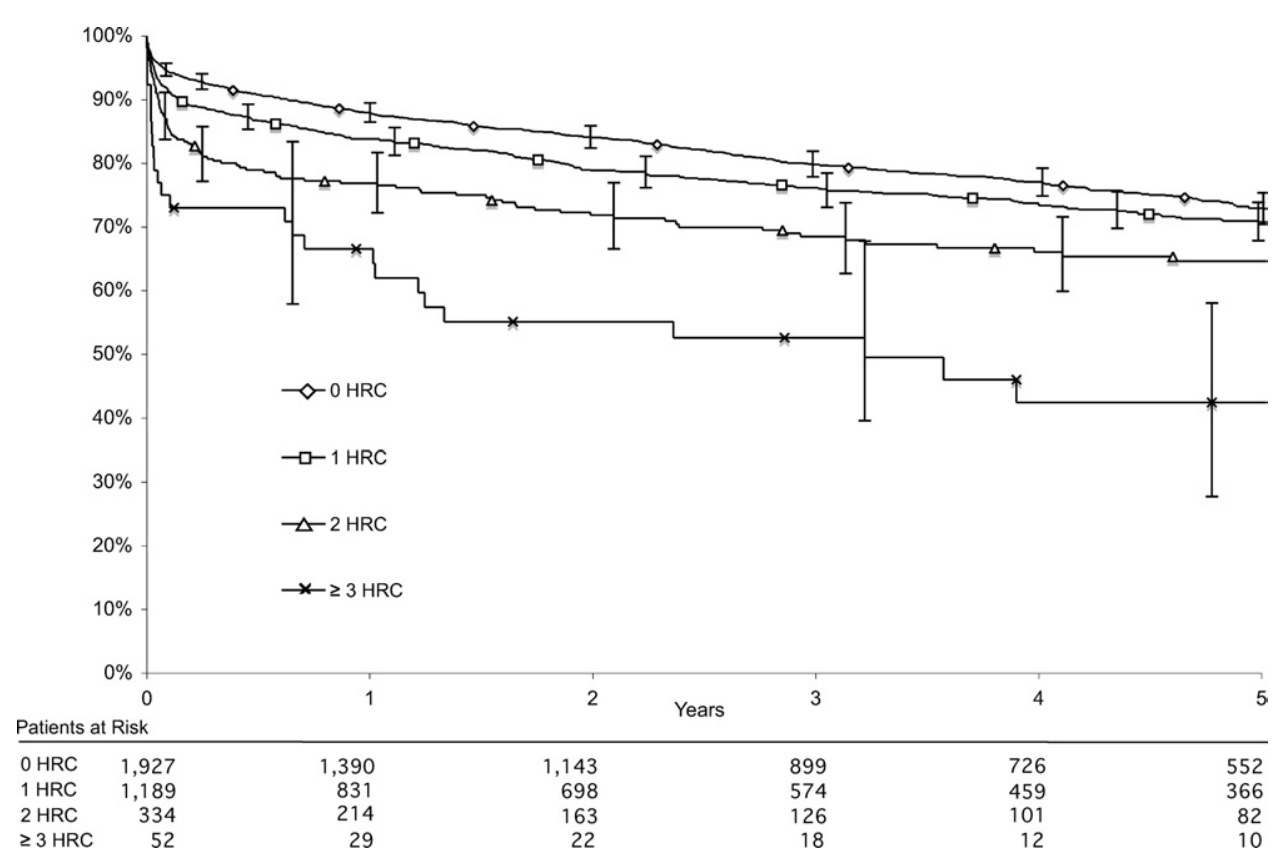

Figure 1. Five-year Kaplan-Meier survival estimates are illustrated as a function of the presence and number of high-risk criteria $(H R C)$ present at the time of transplantation. $(P<.0001$, Wilcoxon test.)

with decreased survival. This is consonant with other studies that have demonstrated the impact of elevated PVR on right ventricular dysfunction, but not on survival. ${ }^{14,15}$ It is likely that current management of right-sided heart dysfunction, including nitric oxide, has largely mitigated its impact on survival. ${ }^{14-17}$ Although this analysis demonstrates a survival advantage to patients with elevated PVR index as well as those undergoing retransplantation, it should be emphasized that this is true in comparison with the high-risk patients and likely reflects a survival in these populations equivalent to the standard risk patients.

Similar negative results were obtained with several other measures of "high-risk" transplant recipients, although the evaluation of hepatitis $\mathrm{C}$ positivity and low donor/recipient weight ratio as risk factors is constrained by the small sample sizes in those populations.

Pretransplant renal failure $(\mathrm{CrCl}$ less than $40 \mathrm{~mL} / \mathrm{min})$ was the only risk factor consistently predictive of poor postoperative survival; the outcomes of patients requiring dialysis (an 8-fold increase in 1-year mortality) reinforce the relationship between renal function and survival. In young patients without intrinsic kidney disease, renal failure may simply act as a surrogate of poorly compensated heart failure. Therefore, these patients should benefit from early mechanical circulatory support and optimization of systemic perfusion.

TABLE 4. Risk factor summation score

\begin{tabular}{|c|c|c|c|c|c|}
\hline \multirow[b]{2}{*}{ Risk factor } & \multicolumn{2}{|c|}{ Thirty-day mortality } & \multicolumn{2}{|c|}{ Contingent 1-year mortality } & \multirow[b]{2}{*}{ Weighting } \\
\hline & $\mathbf{O R}$ & $P$ value & $\mathbf{O R}$ & $P$ value & \\
\hline ECMO & 3.9 & $<.0001$ & 2.1 & .0387 & 4 \\
\hline No. of $H R C \geq 3$ & 2.4 & .0302 & 1.2 & .7116 & 3 \\
\hline Reoperation* & 2.1 & .0105 & 2.8 & .0016 & 2 \\
\hline Stroke* & 1.7 & .2231 & 3.1 & .0053 & 2 \\
\hline Renal failure $(\mathrm{CrCl}<40 \mathrm{~mL} / \mathrm{min})$ & 2.5 & $<.0001$ & 2.1 & $<.0001$ & 2 \\
\hline CHD & 1.6 & .0208 & 1.7 & .0158 & 1 \\
\hline Mechanical ventilation* & 2.0 & $<.0001$ & 1.9 & .0020 & 1 \\
\hline Intensive care unit* & 1.9 & .0008 & 1.7 & .0119 & 1 \\
\hline
\end{tabular}

OR, Odds ratio; ECMO, extracorporeal membrane oxygenation; $H R C$, high-risk criteria; $\mathrm{CrCl}$, creatinine clearance, $C H D$, congenital heart disease. *Weighting based on odds ratio from univariate analysis; see Appendix E1. 


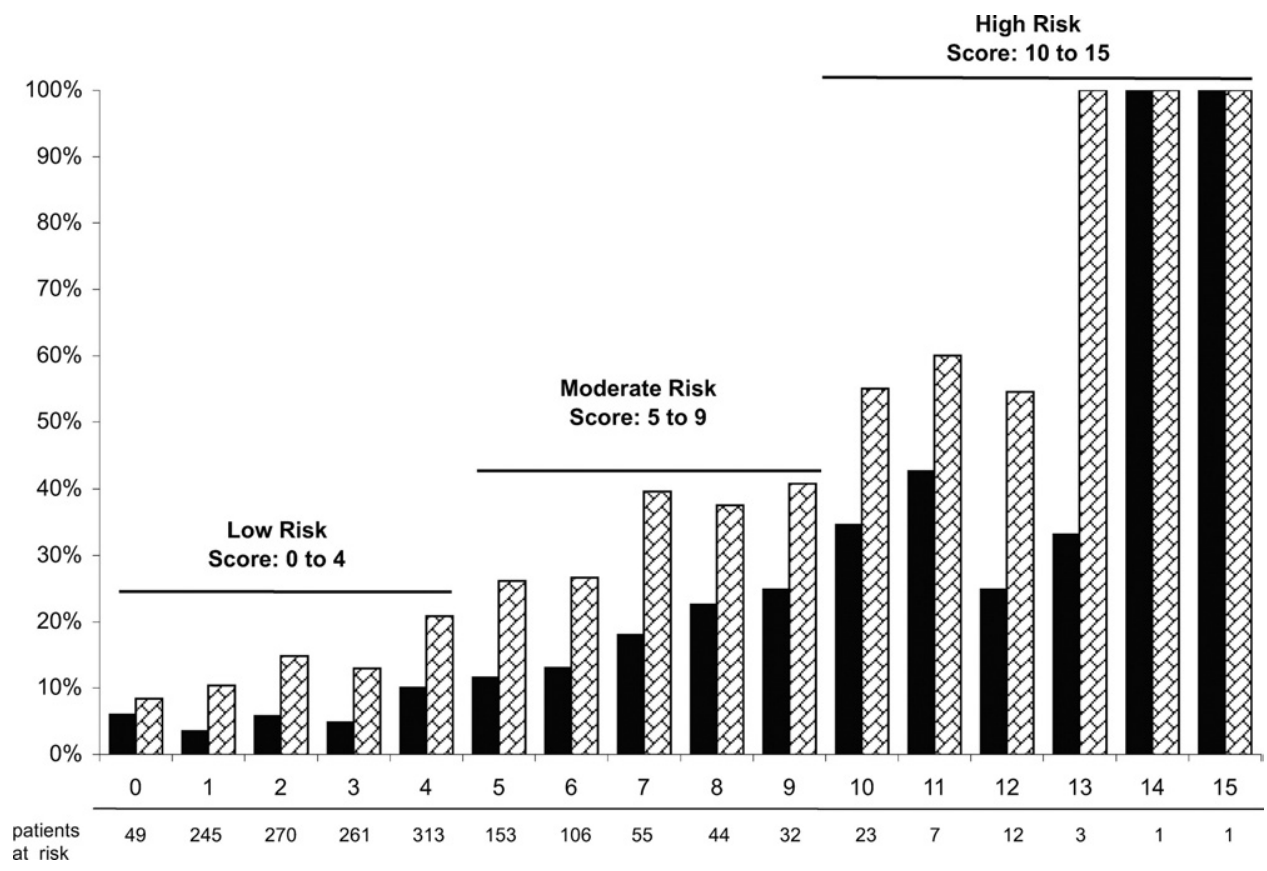

Figure 2. Observed 30-day (black) and 1-year (slanted brick pattern) mortality for each risk score in patients with at least one HRC $(P<0.0001)$.

Because of the usual lack of intrinsic renal disease and the possibility of return of renal function, some have argued that simultaneous renal transplants should be avoided in children. ${ }^{17}$ However, combined transplants have acceptable outcomes in adults and appear to decrease the risk of allograft rejection. ${ }^{18,19}$ Furthermore, simultaneous heart/kidney transplantation may confer a survival advantage over heart transplant alone in patients with dialysis-dependent renal failure. ${ }^{20}$ Sample sizes are limited, but a brief review of 24 simultaneous pediatric

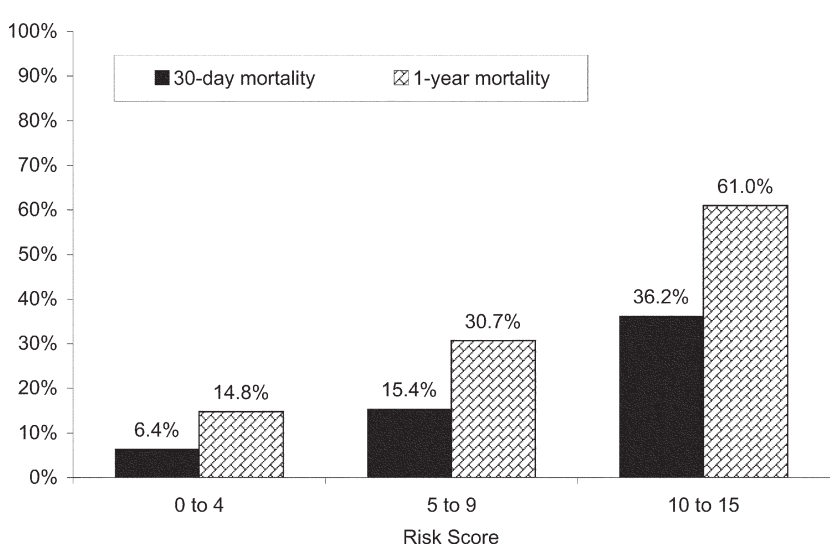

Figure 3. Thirty-day (black) and 1-year (slanted brick pattern) mortality stratified based on risk category (low $=$ score $0-5$, moderate $=6-10$, high $=11-15)$. $P<.0001$. heart/kidney transplantations reported in the UNOS data set suggests that they have similar long-term outcomes with 1-year survival of $85 \%$ (unpublished data); this is supported by case reports providing anecdotal evidence of good long-term outcomes after simultaneous renal transplantation. ${ }^{21,22}$ Further analysis of simultaneous transplants and other therapies for pediatric patients with pretransplant renal dysfunction is warranted. ${ }^{17}$

As noted earlier, we did not include CHD among our HRC because of the perceived limitations in the UNOS database with regard to congenital diagnoses. Despite these limitations, CHD did predict poorer outcomes in this population. Single-institution data suggests that complex CHD accounts for most of this additional risk. ${ }^{10}$ Further studies will be needed in data sets including stratification of congenital diagnoses to accurately elucidate the impact of CHD on posttransplant outcomes.

\section{Impact of Multiple HRC}

Although renal failure was the only one of the traditional HRC to be a significant predictor of early mortality, the accumulation of HRC in a single patient resulted in a significant decrement in early survival. This phenomenon has not previously been described and suggests that criteria for transplantation should include the number of HRC rather than simply the presence or absence of a single risk factor. 


\section{Clinical Status Before Transplant}

In this analysis, as in previous reports, indicators of pretransplant clinical status remain the most significant predictors of poor outcomes. Consistent with previous reports, ${ }^{10,23}$ the need for ECMO was a strong negative predictor of both 30-day and contingent 1-year mortality. The need for hospitalization, especially in the intensive care unit and with mechanical ventilation, was associated with poor outcomes. This (particularly given the negative effects of preoperative or postoperative renal failure) reinforces the need to attempt transplantation before patients becoming critically ill andwhere not possible-to attempt aggressive resuscitation with mechanical ventricular support (rather than ECMO) to improve end-organ function, wean patients from the ventilator, and optimize their clinical condition.

\section{Screening Score for HRC Patients}

The development of a simplified screening score for pediatric patients meeting traditional HRC should provide important prognostic information to physicians, patients, and families as they contemplate transplantation. The scoring system presented here divides patients into three risk groups, with an approximate doubling of mortality between groups: those with scores of 0 to 4 are at low risk (1-year mortality $\sim 15 \%$ ), those with scores from 5 to 9 are at moderate risk (1-year mortality $\sim 30 \%$ ), and those with scores of 10 or more are at high risk (1-year mortality $\sim 62 \%$ ). Although this score applies specifically to patients in the traditional HRC group, further evaluation may enable its applicability to be broadened to a larger cross-section of patients.

Validation of the score may enable its use in accurately stratifying patients into standard and alternate-list recipients. Finally, clinical therapies may be directed at correcting conditions known to predispose to poor outcomes (mechanical ventilation, renal failure, use of ECMO) before transplantation.

\section{Limitations}

These data have several limitations. First, there are problems inherent to the UNOS data set: incomplete data entry and variability between reporting centers as to which pretransplant hemodynamic and clinical variables are reported. Second, although the data analysis supports associations between variables and outcomes, causal relationships cannot be determined. Many of the risk factors may simply be markers for poor clinical status before transplantation rather than a direct causal factor in poor survival. Finally, the scoring system we describe here remains to be validated, and further study is required to confirm its accuracy in predicting early posttransplant outcomes. In addition, it applies specifically to patients within the HRC group and its applicability to standard risk recipients remains to be determined.

\section{Conclusions}

In summary, we have reviewed the UNOS thoracic organ transplant registry and examined the outcomes of patients historically considered at high risk for early transplant mortality. We have found that several of these HRC do not predict poor outcomes but that the cumulative effect of multiple criteria in a single patient results in poor survival. Finally, we have described a simplified screening score to identify the patients within the HRC population most and least likely to survive transplantation. This forms an initial attempt to accurate stratify patients before transplantation. Further efforts are required to optimally allocate the limited supply of donor organs.

We thank UNOS for supplying these data and Katarina Anderson for her assistance with our analysis.

\section{References}

1. Russo MJ, Davies RR, Sorabella RA, Martens TP, George I, Cheema FH, et al. Adult-age donors offer acceptable long-term survival to pediatric heart transplant recipients: an analysis of the United Network of Organ Sharing database. J Thorac Cardiovasc Surg. 2006;132: 1208-12.

2. Chen JM, Russo MJ, Hammond KM, Mancini DM, Kherani AR, Fal $\mathrm{JM}$, et al. Alternate waiting list strategies for heart transplantation maximize donor organ utilization. Ann Thorac Surg. 2005;80:224-8.

3. Laks H, Marelli D, Fonarow GC, Hamilton MA, Ardehali A, Moriguchi JD, et al. Use of two recipient lists for adults requiring heart transplantation. J Thorac Cardiovasc Surg. 2003;125:49-59.

4. Everett JE, Djalilian AR, Kubo SH, Kroshus TJ, Shumway SJ. Heart transplantation for patients over age 60. Clin Transplant. 1996;10:47881.

5. Morgan JA, John R, Weinberg AD, Remoli R, Kherani AR, Vigilance $\mathrm{DW}$, et al. Long-term results of cardiac transplantation in patients 65 years of age and older: a comparative analysis. Ann Thorac Surg. 2003;76:1982-7.

6. Blanche C, Takkenberg J, Nessim S, Cohen M, Czer LS, Matloff JM, et al. Heart transplantation in patients 65 years of age and older: a comparative analysis of 40 patients. Ann Thorac Surg. 1996;62: 1442-6; discussion 1447.

7. Gasink LB, Blumberg EA, Localio AR, Desai SS, Israni AK, Lautenbach $\mathrm{E}$. Hepatitis $\mathrm{C}$ virus seropositivity in organ donors and survival in heart transplant recipients. JAMA. 2006;296:1843-50.

8. McCarthy JF, McCarthy PM, Massad MG, Cook DJ, Smedira NG, Kasirajan V, et al. Risk factors for death after heart transplantation: does a single-center experience correlate with multicenter registries? Ann Thorac Surg. 1998; 65, 1574-8; discussion 1578-9.

9. Chen JM, Davies RR, Mital SR, Mercando ML, Addonizio LJ, Pinney $\mathrm{SP}$, et al. Trends and outcomes in transplantation for complex congenital heart disease: 1984 to 2004. Ann Thorac Surg. 2004;78:135261; discussion 1352-61.

10. Davies RR, Russo MJ, Hong K, O’Byrne ML, Cork DP, Moskowitz AJ, et al. The use of mechanical circulatory support as a bridge-totransplantation in pediatric patients: an analysis of the United Network for Organ Sharing database. J Thorac Cardiovasc Surg 2008; accepted.

11. Canter CE, Shaddy RE, Bernstein D, Hsu DT, Chrisant MR, Kirklin $\mathrm{JK}$, et al. Indications for heart transplantation in pediatric heart disease: a scientific statement from the American Heart Association Council on Cardiovascular Disease in the Young; the Councils on Clinical Cardiology, Cardiovascular Nursing, and Cardiovascular Surgery and 
Anesthesia; and the Quality of Care and Outcomes Research Interdisciplinary Working Group. Circulation. 2007;115:658-76.

12. Lima B, Rajagopal K, Petersen RP, Shah AS, Soule B, Felker GM, et al. Marginal cardiac allografts do not have increased primary graft dysfunction in alternate list transplantation. Circulation. 2006;114(1 Suppl):I27-32.

13. Felker GM, Milano CA, Yager JEE, Hernandez AF, Blue L, Higginbotham MB, et al. Outcomes with an alternate list strategy for heart transplantation. J Heart Lung Transplant. 2005;24:1781-6.

14. Klotz S, Wenzelburger F, Stypmann J, Welp H, Drees G, Schmid C, et al. Reversible pulmonary hypertension in heart transplant candidates: to transplant or not to transplant. Ann Thorac Surg. 2006;82:1770-3.

15. Huang J, Trinkaus K, Huddleston CB, Mendeloff EN, Spray TL, Canter CE. Risk factors for primary graft failure after pediatric cardiac transplantation: importance of recipient and donor characteristics. J Heart Lung Transplant. 2004;23:716-22.

16. Groetzner J, Reichart B, Roemer U, Reichel S, Kozlik-Feldmann R, Tiete A, et al. Cardiac transplantation in pediatric patients: fifteen-year experience of a single center. Ann Thorac Surg. 2005;79:53-60; discussion 61.

17. Sahney S, Chinnock R. Management of infants and young children with combined heart and kidney failure. Pediatr Transplant. 2006;10: 408-12.

18. Groetzner J, Kaczmarek I, Mueller M, Huber S, Deutsch A, Daebritz $\mathrm{S}$, et al. Freedom from graft vessel disease in heart and combined heart- and kidney-transplanted patients treated with tacrolimus-based immunosuppression. J Heart Lung Transplant. 2005;24:1787-92.

19. Trachiotis GD, Vega JD, Johnston TS, Berg A, Whelchel J, Smith AL, et al. Ten-year follow-up in patients with combined heart and kidney transplantation. J Thorac Cardiovasc Surg. 2003;126:2065-71.

20. Russo MJ, Chen JM, Stewart AS, Ascheim DD, Mancini DM, Ratner LE, et al. Long-term survival following combined heart-kidney transplant compared with isolated heart transplant: an analysis of the UNOS database. Transplantation 2006;82(Suppl 3):382.

21. Matteucci MC, Dello Strologo L, Parisi F, Squitieri C, Caione P, Capozza N, et al. Combined heart and kidney transplantation in a child: will we need it more in the future? Transplantation. 1997;63: 1531-3.

22. Kim JJ, Denfield SW, McKenzie ED, Fraser CD Jr, Heinle JS, Goldstein S, et al. Mechanical circulatory support as a bridge to combined dual organ transplantation in children. J Heart Lung Transplant. 2006; 25:1480-2.

23. BarZiv S, McCrindle B, West L, Edgell D, Coles JG, VanArsdell GS, et al. Outcomes of pediatric patients bridged to heart transplantation from ECMO support. ASAIO J. 2007;53:97-102.

\section{Discussion}

David N. Campbell (Denver, Colo). Obviously you and your coauthors have put a lot of work and effort into this very impressive study, which does cover a large number of pediatric and young adults. We actually use 18 and under for pediatric age, so there are some young adults in this "pediatric" group, but 3500 patients is an impressive number of patients over a decade between 1995 and 2005. These were all transplanted and then entered into the UNOS database. Again, this consists of a great deal of time and effort to get this information. I have two comments and then two questions.

As you pointed out well in the manuscript, in pediatric heart transplant recipients, PVR is not an identifier for poor outcome and poor survival as it is in adults. This is something that gets lost sometimes in the pediatric patient group.

You have re-emphasized that the predictors of pretransplant clinical status remain a significant predictor of poor outcomes and, as you have pointed out, these are hospitalization, intensive care unit care, mechanical ventilation, and particularly ECMO support. All of these portend a very poor outcome.
I think the message to be taken from this is that earlier intervention in the pediatric group is warranted, and we are far behind the adults in terms of using ventricular assist devices. I know Columbia, particularly, is a large user of those, and I know that you have already set out a scale very much like you have used for this pediatric group. I think it is very important that the pediatric heart transplant surgeons begin to use mechanical assist devices much earlier. We are obviously coming onto that scene later than we should be. Part is because we do not have good ventricular assist devices in children, but they are being developed and ideally by 2009 , with the money that is being put into five programs, we will have some information and better devices.

My first question to you is, have you actually used this scoring system in any patients to predict outcome, or is this just a scale that you have somewhat arbitrarily determined and are planning on using?

Dr Davies. We have not used it yet. We have developed the score recently and are in the early stages of planning to use it. Importantly, the score needs to be validated before we can use it extensively. This presents a challenge because the UNOS data include all patients who have undergone transplantation in the United States, so it is harder to find patients who are not contained in the database. Our current thinking is to use some Canadian data to validate it, as one of our coauthors is at the University of Toronto. Before that, our intention is to use it as a guide, especially to attempt to reduce the particularly high risk associated with factors like ECMO. The score reinforces the need to wean children from ECMO before transplantation if at all possible. Therefore, at this time, the score is important both to help with prognostication and discussions with families and then to guide clinical therapy in terms of getting people toward a better score before transplantation.

Dr Campbell. My second question concerns a circumstance resembling one of the two examples that you described. We recently performed transplantation in a young child with exactly the circumstances you pointed out. The child had an operation that did not go well, ended up on ECMO for a long period of time, and ended up undergoing transplantation, which in your group would portend again a poor outcome. That child actually did quite well, so it is going to be difficult, I think, to predetermine exactly how these children will do. In our own institution, we were questioned as to whether we should even have undertaken that transplant. My question to you is, what would you do with patients like that in the future who are on say beyond that 10 scoring in your category and are very high risk? Would you just not transplant them? Obviously, part of the idea would be to put them on a ventricular assist device, but even somebody that has been on ECMO for a period of time is not really a good device candidate, as you pointed out. Again, the Columbia group has a scale that they use to determine whether to place devices, and I am not sure some of these patients would even meet the criteria for that. So where do you go with those patients? Do you decide you will not attempt transplantation, or do you go ahead and try?

Dr Davies. Given the lack of validation, it is difficult to say not to transplant them. In addition, although a 50\% 1-year mortality is obviously high, the mortality for the same patient without a transplant is higher. More research needs to clarify which patients will have the most improvement with transplantation and what the best 
method is for optimizing the limited supply of donor organs before we would suggest that this score should be used to exclude people from transplantation.

Dr John Hawkins (Salt Lake City, Utah). I just have a couple of quick questions. How did you pick these risk criteria? How did you pick just seven? Maybe I do not understand the methodology, but why did you not include CHD or multiple previous operations or ECMO as a risk factor in doing the modeling?

Second, have you used this clinically to turn someone down yet? In someone who has three or four or five risk factors, have you actually used this information in your practice?

Dr Davies. To answer your second question first, we have not started using the score yet and the score needs to be validated before its use. We need to refine the accuracy of these data before we can turn people down for transplantation.

With regard to our choice of risk factors, we went through internal Columbia criteria for separating patients who were at standard risk versus high risk for transplant. Most of these criteria were based on consensus statements or older data. In addition, we went through the literature and tried to identify other factors that might be predictive as well.

Specifically, in terms of not including CHD, our own data suggest that patients with CHD as a whole group have similar outcomes and that it is the patients with complex CHD who account for increased risk. Unfortunately, the UNOS data set does not allow one to separate those patients. Therefore, as an a priori decision, we did not want to include it in our list of criteria.
However, it did turn out to be a reliable risk factor even in the UNOS data set, so it was included in our risk score based on predetermined methodology. Other information provides significant opportunity for improvement: either single institution studies or the Pediatric Heart Transplant Study Group, which includes much more detailed diagnosis information that will allow us to tease out the true contribution of CHD to posttransplant outcomes.

Dr Jonathan Chen (New York, NY). I just want to make a comment. One big impetus for us to pursue this question at all is that we lag behind the adult service mainly because the numbers are so small. We have had a lot of success in the adult arena with an alternate list comprised of a separate group of patients with whom we have a very stern conversation pretransplant indicating that they are not candidates in the routine pool but could be candidates on the alternate list made for patients who are really high risk. I believe it does make clear for families and for the patients themselves the gravity of the situation. What we did find just anecdotally in the adult world with the alternate list (these are organs that are passed over by every other program) is that about $50 \%$ of them end up being normal hearts. They just do not have the appropriate size to recipient available. This is particularly pertinent for the pediatric group. As I am sure everyone knows, you will often get a telephone call about a young infant donor and everybody has passed it over because it is simply not the right size. It is not that these patients get "marginal hearts" and thereby stack donor variables with recipient variables. I think it gives them a good shot at transplant, albeit at high risk. 


\section{Appendix E1}

All significant multivariate predictors of 30-day mortality were included in the score. Significant univariate predictors were also included when certain criteria were met: (1) they were highly predictive of mortality but the variable was not coded in a sufficient number of patients to meet the threshold $P$ value for entry into the multivariate model and (2) inclusion in the scoring scale increased the predictive value of the scale (measured by receiver operating curves, c-score, and $\chi^{2}$ tests. The score assigned to each risk factor (given in Table 4) was based on the OR for 30-day and 1 -year mortality and was adjusted to maximize the predictive value of the scoring scale at both time periods. 


\section{TABLE E1. List of analyzed variables}

Year of transplant

Age (both continuous and stratified)

Weight (both continuous and stratified)

Body surface area (both continuous and stratified)

Etiology: congenital heart disease

Etiology: dilated cardiomyopathy

Etiology: retransplant

Ventricular assist device at transplantation

Extracorporeal membrane oxygenation at transplantation

Intra-aortic balloon pump at transplantation

Any type of mechanical circulatory support at transplantation

Need for inotropic support at transplantation

Need for mechanical ventilation at transplantation

Hospitalized at transplantation

In intensive care unit at transplantation

Requirement for transfusions between listing and transplantation Pulmonary vascular resistance index $>6$ Wood units

History of chronic renal failure

Renal failure as indicated by creatinine clearance $<40 \mathrm{~mL} / \mathrm{min}$

History of stroke

History of pulmonary embolus

History of previous cardiac surgery

Prolonged ischemic time ( $>4$ hours)

Hepatitis C positivity

Low donor/recipient weight ratio $(<0.7)$

Elevated panel reactive antibodies (both $>10 \%$ and $>40 \%$ )

Presence of high-risk criteria

Number of high-risk criteria present

Gender

Waiting time

Days of waiting as status $1 / 1 \mathrm{~A}$

Percentage of time waiting as status $1 / 1 \mathrm{~A}$

Cardiac output before transplantation

Donor age

Allograft ischemic time

Donor creatinine

Donor/recipient weight ratio

Pulmonary vascular resistance (continuous variable)
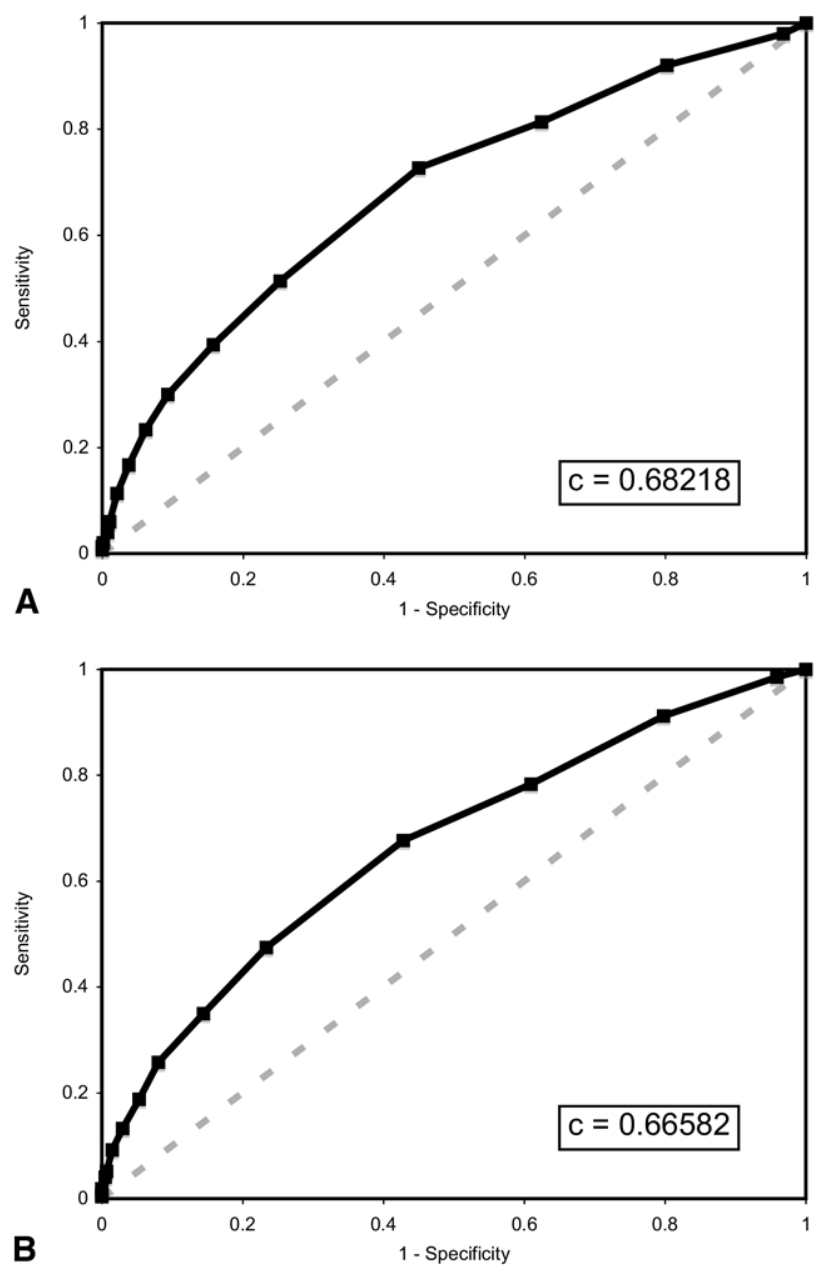

Figure E1. Receiver operating curves are shown for both 30-day (A) and overall 1-year survival (B) based on the scoring scale. In both cases, as demonstrated by the $c$ score, the scoring scale adequately predicts survival. 